\title{
Kommentar zu „Green IT: Ein Thema für die Wirtschaftsinformatik?"
}

\section{DOI 10.1007/s11576-011-0300-4}

\section{Der Autor}

Prof. Dr. Andreas Gadatsch ( $\varangle$ )

Hochschule Bonn-Rhein-Sieg

Professur für

Betriebswirtschaftslehre, insb.

Wirtschaftsinformatik

Grantham-Allee 20

53757 Sank Augustin

Deutschland

Andreas.Gadatsch@h-brs.de

Online publiziert: 2011-10-28

This article is also available in English via http://www.springerlink.com and http://www.bise-journal.org: Loos, P, Gadatsch A (2011) Comments on "Green IT: A Matter of Business and Information Systems Engineering?". Bus Inf Syst Eng. doi: 10.1007/ s12599-011-0190-4.

(C) Gabler Verlag 2011

\section{Green IT und IT-Controlling}

Im Heft 4/2011 stellte Peter Loos mit weiteren Autoren dankenswerter Weise die Frage „Green IT: Ein Thema für die Wirtschaftsinformatik?"zur Diskussion (Loos et al. 2011). Diese Thematik haben wir in den letzten Jahren aus dem Blickwinkel des IT-Controllings untersucht.

Die gestiegenen Kosten für Energie haben im IT-Management zu einem Um- denken geführt. Unter dem Stichwort „Green IT“ initiieren viele Unternehmen Maßnahmen, um Ihren Energieeinsatz und damit die Kosten für den IT-Betrieb zu senken. Teilweise hat dies sogar zu neuen Berufsbildern wie den „Chief Sustainability Officer" geführt, der die Maßnahmen im Unternehmen bündeln soll. Nach einer Untersuchung der Deutschen Bank Research haben $38 \%$ der Unternehmen bereits einen „Green-ITBeauftragten" berufen (Deutsche Bank Research 2011, S. 5).

In zwei Umfragen (2009 und 2011) unter IT-Controllern und IT-Führungskräften (CIO u.a.) haben wir die Verbreitung von Green-IT-Maßnahmen und den damit bisher erreichten Erfolg sowie die Zukunftspläne der Unternehmen untersucht (Gadatsch und Juszczak 2009, 2011). Im Rahmen unserer Erhebungen konnten wir feststellen, dass bei etwa $60 \%$ der Unternehmen Maßnahmen im Umfeld Green IT initiiert wurden, meist vom jeweiligen CIO, zum Teil aber auch von der Unternehmensleitung. Zudem beschäftigen sich nach der aktuellen Umfrage in größeren Unternehmen IT-Controller verstärkt mit Green IT, i. d. R. um die Kosteneinsparpotenziale zu realisieren. Obwohl bereits insbesondere im Umfeld des Rechenzentrummanagements (Kühlung, Gebäudetechnik) und Softwaremanagements (CloudComputing) große Erfolge erzielt wurden, besteht nach wie vor Handlungsbedarf, weil die Unternehmen nicht immer mit den erzielten Ergebnissen zufrieden sind.
Für die Forschung gibt es nach unserer Meinung noch deutlichen Handlungsbedarf in den Themenfeldern Zertifizierung und Reifegradermittlung. Nur $7 \%$ der befragten Unternehmen gaben beispielsweise an Methoden zur „GreenIT-Reifegradermittlung“ einzusetzen. Vielen Unternehmen (21 \%) war das Thema überhaupt nicht bekannt. Ähnlich sieht das Bild bei der „Green-ITZertifizierung“ aus. Hier dominiert derzeit der „TÜV“ den „Green-IT-Zertifizierungsmarkt“.

Fazit: Nachhaltigkeit und Green IT sind wichtige Themen für das ITControlling und damit auch die Wirtschaftsinformatik.

\section{Literatur}

Deutsche Bank Research (2011) (Hrsg) Green IT, more than a passing fad! Deutsche Bank, Frankfurt. http://www.dbresearch.com. Abruf am 2011-01-13

Gadatsch A, Juszczak J (2011) Ergebnisse der Kurzumfrage zum Stand von Green IT im deutschsprachigen Raum 2011. In: Schriftenreihe des Fachbereiches Wirtschaftswissenschaft Sankt Augustin, Bd 30. Hochschule Bonn-Rhein-Sieg, Sankt Augustin

Gadatsch A, Juszczak J (2009) Ergebnisse der Kurzumfrage zum Stand von Green IT im deutschsprachigen Raum (Green IT 2009). In: Schriftenreihe des Fachbereiches Wirtschaftswissenschaft Sankt Augustin, Bd 24. Hochschule Bonn-Rhein-Sieg, Sankt Augustin

Loos P, Nebel W, Marx Gomez J, Hasan $H$, Watson RT, vom Brocke J, Seidel S, Recker J (2011) Green IT: Ein Thema für die Wirtschaftsinformatik? BISE 3(4):245-252 